\title{
If You Have a Low Adenoma Detection Rate, Don't Blame Your Fellows
}

\author{
Dayna S. Early
}

Published online: 10 October 2013

(c) Springer Science+Business Media New York 2013

Quality measures in colonoscopy are at the forefront in the field of gastroenterology, particularly in light of the current emphasis on "accountable" care. Colonoscopy quality measures include cecal intubation rate, complication rate, adenoma detection rate (ADR), and appropriate screening and surveillance intervals. For screening and surveillance colonoscopy, ADR is emerging as perhaps the most important quality indicator, since identification and removal of adenomas is the primary goal. Factors that improve ADR include adequacy of bowel preparation, cecal withdrawal time, time of day that colonoscopy is performed, type of sedation regimen used, and use of techniques such as cap-assisted colonoscopy and retroflexion in the right colon $[1,2]$.

Whether fellow involvement in colonoscopy affects ADR, the subject of the paper by Oh et al. [3] in this issue of Digestive Diseases and Sciences, has been less well studied. Most endoscopists who train fellows to perform colonoscopy probably have an opinion, based on personal experience, about whether fellow involvement increases or decreases ADR. Oh et al. looked at the issue more objectively by performing a meta-analysis of relevant studies to determine if a difference in polyp detection rate (PDR) or ADR occurs when a fellow is involved in colonoscopy. On the one hand, it might seem intuitive that PDRs and ADRs would be higher with a fellow and attending both observing the same procedure, as dual observers increase ADR [4]. Additionally, since fellows tend to take longer to complete a colonoscopy, one might infer that increased cecal

\section{S. Early $(\bowtie)$}

Department of Medicine, Washington University in St. Louis, 660 South Euclid Avenue, Campus Box 8124, St. Louis, MO 63110, USA

e-mail: dearly@dom.wustl.edu withdrawal time by a fellow is associated with an increased ADR, similar to the data showing increased ADR with increased cecal withdrawal time amongst attending endoscopists. On the other hand, one might predict a lower ADR with fellows due to other factors, such as rapid withdrawal through colon segments when the fellow is having difficulty managing the colonoscope, a less thorough examination of the back surfaces of colonic folds, and inability to retroflex the colonoscope in the right colon.

The impact, if any, of fellow participation on the PDR and ADR is important information for clinicians who regularly train fellows, particularly if ADR is recorded and/ or reported as a quality indicator. Although PDR is not relevant for determining screening and surveillance intervals, calculating PDR is much simpler since polyp histology is not required. If PDR correlates with ADR, some programs may choose to monitor PDR as a useful metric as fellows progress through training, allowing an objective assessment of fellow proficiency. Additionally, there may be patients who receive care at training institutions who want to know whether having a trainee involved affects the quality of their medical care.

To determine if fellow involvement in colonoscopy affects ADR, Oh et al. [3] performed a comprehensive literature search, identifying articles in which the ADR, as well as the percent of colonoscopies, involving fellows was reported. From this information, they applied a modified Quality Assessment of Diagnostic Accuracy Studies (QUADAS) tool to the data to determine the impact of fellow participation in colonoscopy on ADR. A total of 14 studies met inclusion criteria, accounting for 21,504 colonoscopies. Based on the combined data, the RR (95\% CI) for detecting polyps when a fellow was present was 1.04 $(0.94,1.15)$, and for detecting adenomas was 1.03 (0.93, 1.14) suggesting that fellow involvement has no impact on 
PDR or ADR. There was no affect of age or gender on ADR between the fellow plus attending versus attending only colonoscopies, with similar percentages of patients reported as having poor bowel preparation. A strength of this meta-analysis is the large number of colonoscopies analyzed, which likely narrowed the confidence intervals. The overall ADR was $30.8 \%$, which is slightly higher than the accepted rate of $15 \%$ for women and $25 \%$ for men [5]. This suggests that the colonoscopies in the studies are representative of a typical screening and surveillance population, and that the fellows and attendings were appropriately vigilant in their mucosal inspection.

Still, there are important limitations to this meta-analysis that leave us wondering if fellow involvement in colonoscopy actually impacts PDR or ADR. Discerning the role of fellow involvement on PDR and ADR was not the primary outcome of some studies, limiting raw data interpretability, bringing to question the validity of the calculated confidence intervals for the meta-analysis. Importantly, none of the studies randomized patients to a fellow plus attending physician colonoscopy versus an attending physician only colonoscopy. Even though the characteristics of the patients did not appear different, it is impossible to distinguish from a retrospective meta-analysis if there were any important differences in adenoma risk factors between fellow plus attending and attending only patients. Secondly, the degree of fellow involvement was not reported in any of the 14 studies, so it remains unclear if the mucosal inspection is more representative of the fellow's technique or the attending physician's technique. As an example, since colonoscope insertion is typically more challenging than colonoscope withdrawal, even inexperienced fellows may participate in the latter but not in the former. Most of the studies did not report the level of training of the fellow, which has been shown in some studies, but not others, to correlate with higher ADR [6-8]. Finally, three studies reported PDR but not ADR, which limits the conclusions one can draw from these studies [9$11]$.

Since detecting and removing adenomas is the goal of screening and surveillance colonoscopy, it is the responsibility of the attending physician to ensure that optimal mucosal inspection is performed. Fortunately, Oh et al. did not report lower ADR with fellow involvement, since this would imply that the attending was in some way distracted or impaired by a fellow's presence. Similarly, it is reassuring that $\mathrm{Oh}$ et al. did not report higher ADR with fellow involvement, since this would imply that attending endoscopists are underperforming when fellows are not present. When supervising fellows, attending physicians have two main responsibilities; first delivering high-quality colonoscopy to our patients, and second, teaching our fellows how to become skilled in delivering high-quality colonoscopy. In the future, it is likely that ADRs will be expected to be even higher than referenced above as endoscopic technology improves, combined with increasing emphasis on endoscopic quality.

In summary, the study by Oh et al. suggests that fellow participation is "quality-neutral" with regard to ADR. We should be cautious and avoid interpreting these results as evidence that fellows are just as good as attendings at detecting polyps. Experienced fellows may be very good at adenoma detection, but attending endoscopists must remain vigilant during colonoscopy when fellows are involved. In fact, our role is to teach the fellows the skills to perform a careful mucosal inspection and to emphasize the importance of maximizing adenoma detection, thus affirming the overall impression that the care delivered at teaching institutions is superior or at least equal to the care delivered in the community.

\section{References}

1. Rastogi A, Bansal A, Rao DS, et al. Higher adenoma detection rates with cap-assisted colonoscopy: a randomised controlled trial. Gut. 2012;61:402-408.

2. Hewett DG, Rex DK. Miss rate of right-sided colon examination during colonoscopy defined by retroflexion: an observational study. Gastrointest Endosc. 2011;74:246-252.

3. Oh, YS, Collins CL, Virani S, et al. Lack of impact on polyp detection by fellow involvement during colonoscopy: a metaanalysis. Dig Dis Sci. (Epub ahead of print). doi:10.1007/s10620013-2701-2.

4. Lee CK, Park DI, Lee SH, et al. Participation by experienced endoscopy nurses increases the detection rate of colon polyps during a screening colonoscopy: a multicenter, prospective, randomized study. Gastrointest Endosc. 2011;74:1094-1102.

5. Rex DK, Petrini JL, Baron TH, et al. Quality indicators for colonoscopy. Am J Gastroenterol. 2006;101:873-885.

6. Peters SL, Hasan AG, Jacobson NB, Austin GL. Level of fellowship training increases adenoma detection rates. Clin Gastroenterol Hepatol. 2010;8:439-442.

7. Buchner AM, Shahid MW, Heckman MG, et al. Trainee participation is associated with increased small adenoma detection. Gastrointest Endosc. 2011;73:1223-1231.

8. Rogart JN, Siddiqui UD, Jamidar PA, Aslanian HR. Fellow involvement may increase adenoma detection rates during colonoscopy. Am J Gastroenterol. 2008;103:2841-2846.

9. Kaneshiro M, Ho A, Chan M, Cohen H, Spiegel BM. Colonoscopy yields fewer polyps as the day progresses despite using social influence theory to reverse the trend. Gastrointest Endosc. 2010;72:1233-1240.

10. Shah N, Sanaka MR, Mullen KD, Amini S, McCullough AJ. Comparison of polyp detection rates during colonoscopy by attending staff along versus trainee plus attending staff: are two people better than one? Gastrointest Endosc. 2005;61:AB264.

11. Tee HP, Corte C, Al-Ghamdi H, et al. Prospective randomized controlled trial evaluating cap-assisted colonoscopy vs standard colonoscopy. World J Gastroenterol. 2010;16:3905-3910. 